This document is the unedited Author's version of a Submitted Work that was subsequently accepted for publication in Langmuir, copyright $\odot$ American Chemical Society after peer review. To access the final edited and published work see: https://dx.doi. org/10.1021/acs. langmuir.8b00162. 


\section{Lipid monolayer formation and lipid exchange}

\section{monitored by a graphene field-effect transistor}

Benno M. Blaschke ${ }^{1 *}$, Philip Böhm ${ }^{2,3 *}$, Simon Drieschner ${ }^{1}$, Bert Nickel ${ }^{2,3}$, Jose A. Garrido ${ }^{4,5}$

${ }^{1}$ Walter Schottky Institut und Physik-Department, Technische Universität München, Am Coulombwall 4, 85748 Garching, Germany

${ }^{2}$ Fakultät für Physik and Center for NanoScience, Ludwig-Maximilians-Universität, Geschwister-Scholl-Platz 1, 80539 München, Germany

${ }^{3}$ Nanosystems Initiative Munich, Schellingstraße 4, 80799 München, Germany

${ }^{4}$ Catalan Institute of Nanoscience and Nanotechnology (ICN2), CSIC and The Barcelona Institute of Science and Technology, Campus UAB, Bellaterra, 08193 Barcelona, Spain

${ }^{5}$ ICREA, Pg. Lluís Companys 23, 08010 Barcelona, Spain

${ }^{*}$ These authors contributed equally. 


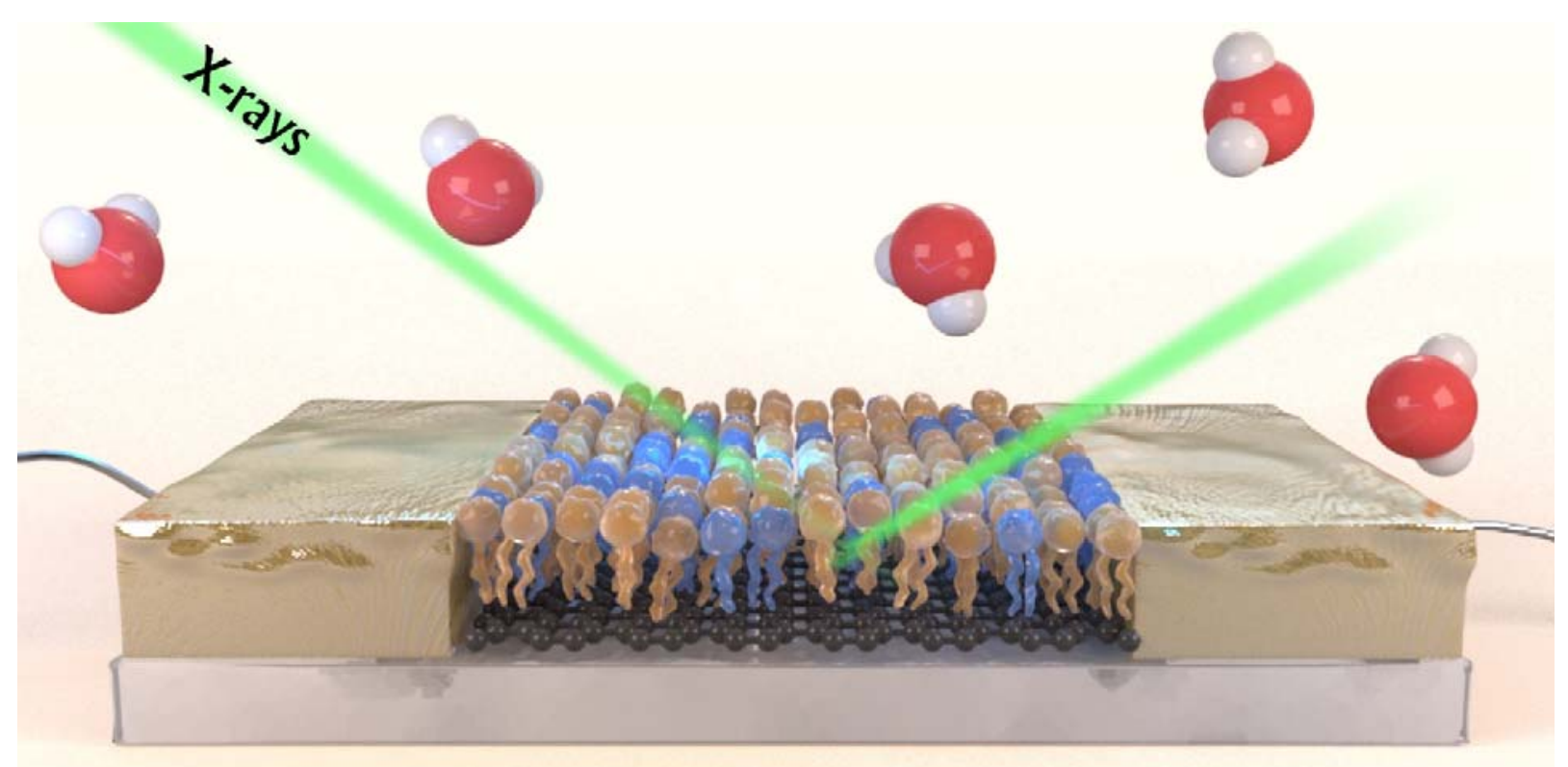

KEYWORDS: Graphene, Transistor, Biosensing, X-ray reflectometry, Lipid

\section{ABSTRACT}

Anionic and cationic lipids are key molecules involved in many cellular processes; their distribution in biomembranes is highly asymmetric and their concentration is well controlled. Solution-gated graphene field-effect transistors (SGFETs) exhibit high sensitivity towards the presence of surface charges. Here, we establish conditions that allow the observation of the formation of charged lipid layers on solution-gated field-effect transistors in real time. We quantify the electrostatic screening of electrolyte ions and derive a model that explains the influence of charged lipids on the ion sensitivity of graphene SGFETs. The electrostatic model is validated using structural information from X-ray reflectometry measurements, which show that lipid monolayer forms on graphene. We demonstrate that SGFETs can be used to detect cationic lipids by self-exchange of lipids. Furthermore, SGFETs allow measuring the kinetics of layer formation induced by vesicle fusion or spreading from a reservoir. Due to the high transconductance and low noise of the electrical readout, we can observe characteristic 
conductance spikes that we attribute to bouncing-off events of lipid aggregates from the SGFET surface, suggesting a great potential of graphene SGFETs to measure the on-off kinetics of small aggregates interacting with supported layers.

\section{INTRODUCTION}

Graphene solution-gated field-effect transistors (SGFETs) have received increased attention in the field of biosensing during the last years. Their high transconductance and low intrinsic electronic noise result in high signal-to-noise ratio (SNR) ${ }^{1}$. Additionally, they provide excellent time resolution ${ }^{2,3}$ operating up to the $\mathrm{MHz}$ regime. Due to their stability in electrolyte environments $^{4,5}$ and established surface functionalization schemes ${ }^{6}$, they represent a promising biosensing platform. Indeed, SGFETs have been used for recording of cell action potential ${ }^{7-10}$ and brain activity ${ }^{11,12}$, and for the detection of analytes such as neuro transmitters ${ }^{13}, \mathrm{DNA}^{3,14}$, and prostate specific antigen ${ }^{15}$. Graphene SGFETs also hold great potential to study supported lipid membranes ${ }^{16}$, i.e., low dimensional fluids ${ }^{17,18}$ used to build model cells ${ }^{19,20}$, to study ion channel activity $^{21}$, and to design highly selective biosensors ${ }^{22}$.

Here, we address the question how to detect charged lipids. Charged lipids are of specific interest since they play a crucial role in cell signaling, the formation of functional domains as well all as the arrangement of membrane proteins and therefore, e.g., as an important marker for $\operatorname{apoptosis}^{23}$

Optical microscopy is per se insensitive to charged lipids; thus fluorescence techniques rely on labeling techniques, i.e., phosphoserine lipids (PS) can be imaged by labeling kits using binding of fluorescent annexins to PS via divalent $\mathrm{Ca}^{2+}$ ions ${ }^{24}$. Since these binding assays passivate the PS, there is a need for a label-free electrical readout. Label-free detection reduces both complexity and cost of the experiments, as well as it excludes any possible influence of a dye on 
the outcome of a measurement. Solution-gated transistors are well suited for this application if the transconductance $g_{m}$ is high. For SGFET devices $g_{m}$ is determined by the charge carrier mobility, the capacitance of the interface with the electrolyte, and the device dimensions. Despite their proven applicability for biosensing ${ }^{21-23}$ organic field-effect transistors mostly have low mobility, up to several dozen $\mathrm{cm}^{2} / \mathrm{Vs}^{24}$ in best cases. Some organic materials, in particular PEDOT:PSS, show a huge effective capacitance, which compensates the low mobility at the cost of the response time ${ }^{25}$, which is limited to the $\mathrm{kHz}$ region and requires carefully designed geometries in order to achieve ms resolution ${ }^{26,27}$. Lipid membranes on PEDOT:PSS hinder ion diffusion which further slow down the performance of PEDOT:PSS based transistors ${ }^{28}$. Another drawback is the rough structure of the polymer which also swells upon water immersion ${ }^{28}$. For more classical semiconductors such as gallium nitride and silicon, the interface capacitance is low due to the required use of thin dielectric layers separating the semiconductor from the electrolyte, which significantly reduce the semiconductor/electrolyte capacitance ${ }^{1,29}$. Surface conductive diamond needs no insulation layer, provides decent mobility but the lipid membranes were shown to degrade $g_{m}$ significantly ${ }^{30,31}$. Nanowire and carbon nanotube transistors provide smooth surfaces and high transconductance and sensitivity ${ }^{32-36}$. The effect of lipid membranes on the electrical properties of the nanowires and transport through lipid membranes has been previously studied ${ }^{37-39}$. Monitoring of lipid layer formation is possible but evidently limited to a very small $\operatorname{area}^{40}$.

Besides exhibiting high transconductance $\mathrm{g}_{\mathrm{m}}$ and low noise, graphene transistors provide sensing capabilities on larger areas and have a very flat surface. Due to its ultimate surface to volume ratio, graphene is also very sensitive to charged lipid head groups of a lipid layer in its vicinity. The high temporal resolution $<1 \mu$ s of graphene SGFETs $^{41}$ is comparable to the time 
resolution of fluorescence correlation spectroscopy (FCS) and could enable the electrical detection of protein interaction with lipid membranes. Although several studies on lipid layers on graphene were published, key questions such as the lipid structure on the hydrophobic graphene are still under debate. Both lipid monolayer and bilayer formation have been reported ${ }^{42-45}$. In addition, none of the published studies used graphene SGFETs to monitor the formation of lipid layers, a key question of fundamental interest ${ }^{46}$. For instance, SGFETs could allow the study of single vesicle adsorption and spreading. This label-free detection of single lipid exosomes adsorption and spreading is of great interest for cancer diagnostics ${ }^{47}$.

Here, we use impedance spectroscopy and X-ray reflectometry to clarify the structure of lipid monolayers on graphene. Next, we study electrostatic interactions of lipid layers with graphene transistors in detail. We investigate the influence of the lipids on the ion sensitivity of the transistors, explain the changes in ion sensitivity and estimate the surface charge density of the lipid membrane. Afterwards, we use graphene transistors to study the formation of lipid layers for different preparation techniques, from micro to macro scale, and provide insights into the fusiogenity of lipid vesicles.

\section{RESULTS AND DISCUSSION}

Supported lipid layers were deposited on graphene by vesicle fusion, and by stamping and subsequent spreading ${ }^{48}$ (see methods section for details). The standard characterization of the layer formation by fluorescence microscopy is not possible, i.e., we find that fluorescently labeled lipids remain dark. Apparently, the fluorescence of the dyes is quenched, as expected for a surface with metallic character ${ }^{49}$. We therefore speculate that fluorescence measurements of fluorescently labeled lipid layers on graphene ${ }^{42,43}$ reported in very few reports could be related to 
transfer residues, which increase the separation between the fluorescence dye and the graphene, thus reducing the quenching effect ${ }^{45,50}$.

On hydrophobic substrates, lipids tend to form monolayers ${ }^{51,52}$. For graphene, recent measurements also suggest the formation a lipid monolayer, based on quartz crystal micro balance experiments ${ }^{44}$. To provide direct structural evidence for the lipid monolayer on graphene, we have performed X-ray reflectometry (XRR) measurements. Reflectometry allows to analyze the structure of lipid layers on more and more complex interface structures ${ }^{53-58}$. Reflectometry probes areas of up to several square centimeters due to the large illumination spot by the incoming beam at low angles of incidence. The specular reflection of X-rays contains information about the scattering length density (SLD) profile normal to a surface ${ }^{59,60}$. The $q$ range covered in the measurement extends up to $\mathrm{q}_{\max } \approx 0.5 \AA^{-1}$, which implies that the scattering length density distribution can be decomposed with a resolution of approximately $6 \AA$ according to Fourier sampling theory ${ }^{60}$.

In order to disentangle the different surface layers, we compare XRR measurements on the bare $\mathrm{Si}$ substrate, including its $\mathrm{SiO}_{2}$ layer of around $200 \mathrm{~nm}$, with the same sample after graphene transfer and after formation of a 1,2-dioleoyl-3-trimethylammonium-propane (DOTAP) layer by vesicle fusion (see experimental methods for details). All three measurements were performed in Dulbecco's PBS buffer (ionic strength $165 \mathrm{mM}$ ). Performing these consecutive experiments is essential to provide proper reference measurements that allow for modelling of the X-ray intensities due to the stratified $\mathrm{SiO}_{2} /$ graphene/lipid layer structure. All reflectivity data are shown as grey rectangles in Figure 1a. After the graphene transfer, the X-ray reflectivity curve shows a significant difference compared to the bare $\mathrm{SiO}_{2}$ substrate, as seen in the inset of Figure 1a. This 
underlines the sensitivity of the reflectometry measurements towards the graphene transfer. After depositing the lipids, an even stronger change indicates the formation of a lipid layer. The SLD profiles were modeled using MOTOFIT ${ }^{61}$; see experimental methods for a description of the fitting procedure. The modelling yields the values of the thickness, roughness and SLD value of each layer. Models were iteratively applied to all three reflectivity curves to identify a consistent structure model in accordance with all three data sets (bare substrate with $200 \mathrm{~nm}$ layer of $\mathrm{SiO}_{2}$, after the graphene transfer and after lipid deposition). The best fits (solid lines in Figure 1a) are all in good agreement with the experimental data.

The SLD profiles are shown in Figure 1b. For the bare wafer, the fit reveals a roughness of $4.05 \AA$ for the $200 \mathrm{~nm}$ thick oxide layer while the SLD of $\mathrm{SiO}_{2}$ is $18.7 * 10^{-6} \AA^{-2}$. The data recorded after the graphene transfer can be reproduced by one additional layer. Its thickness was determined to be $12.59 \AA$ with a roughness of $1.11 \AA$ (in good agreement with AFM measurements ${ }^{62,63}$ ) and an SLD of $8.2 * 10^{-6} \AA^{-2}$. These values indicate that the graphene is not entirely flat and might contain transfer residues ${ }^{63}$. Further parameters used for modelling the data can be found in Table S1 in the supplementary information.

In order to model the reflectometry data of the DOTAP layer, three layers on top of the silicon $/ \mathrm{SiO}_{2}$ substrate were needed. In the modelling, a lipid monolayer requires one layer for the lipid tails next to one layer for the lipid head groups. Densely packed lipid tails have an SLD lower than the SLD of water $\left(9.45^{*} 10^{-6} \AA^{-2}\right)$. The lipid head groups have an SLD higher than water $^{64}$. Note that the actual SLD, i.e. the electron density, of lipid tails and head groups varies linearly with the packing density of the lipids and the system's roughness. Only models where the hydrophilic lipid head groups face towards the buffer were considered. The SLD of water is 
shown as a reference (dashed line) in Figure 1b. After the deposition of the lipid layer the thickness of the layer adjacent to the silicon oxide was fitted to be $12.66 \AA$ with a roughness of $1.11 \AA$ and an SLD of $8.28^{*} 10^{-6} \AA^{-2}$. This is in good agreement with the values of the graphene layer of the measurement without lipids. Next to the graphene, a second layer with a thickness of $9.92 \AA$, a roughness of $4.47 \AA$ and an SLD of $7.99 * 10^{-6} \AA^{-2}$ and a third layer with a thickness of $10.54 \AA$, a roughness of $2.71 \AA$ and an SLD of $9.93 * 10^{-6} \AA^{-2}$ is present. The latter layers represent lipid tails and heads, respectively. Thus, the XRR experiments confirm the formation of a $20.5 \AA$ thick lipid monolayer, on graphene.

In order to provide further evidence on the lipid monolayer structure on graphene, we have performed spreading experiments on hydrophilic $\mathrm{SiO}_{2}$ patterned with graphene barrier structures. It has been shown in previous work that lipid bilayers form on the hydrophilic silicon dioxide $^{51,65}$. Since spreading lipid bilayers do not cross hydrophobic/hydrophilic borders ${ }^{18,51}$, probing the spreading behaviour on the $\mathrm{SiO}_{2}$ /graphene interface can give further insight into the lipid layer formation on graphene. The first panel in Figure 1c shows a schematic of the experiment. DOTAP lipids were stamped on a silicon dioxide surface. Graphene domains forming rectangular obstacles are located below. The bold black regions in panel 1 of Figure 1c correspond to the graphene domains and the inner white regions correspond to the $\mathrm{SiO}_{2}$ domains. After addition of buffer a lipid bilayer spreads across the substrate ${ }^{51}$. In Figure 1c, panels (2) to (4) show fluorescence images of the spreading process. First, the lipid bilayer spreads evenly across the $\mathrm{SiO}_{2}$ surface (2), as observed previously ${ }^{18}$. The lipids also spread through the $\mathrm{SiO}_{2}$ channels formed in between the graphene obstacles, leading to a curved lipid front (3). Finally, the lipid front leaves the graphene obstacles behind; a continuous straight lipid front is visible again (4). Since no fluorescence was observed in the inner regions of the graphene domains, 
where a $\mathrm{SiO}_{2}$ surface is exposed, we can conclude that the bilayer lipids do not spread across the hydrophilic/hydrophobic graphene/silicon dioxide border in agreement with previous reports ${ }^{66}$. The spreading results summarized in Figure 1c underline the hydrophobicity of graphene structures on silicon dioxide ${ }^{67}$ and support the finding that a lipid monolayer forms on graphene.
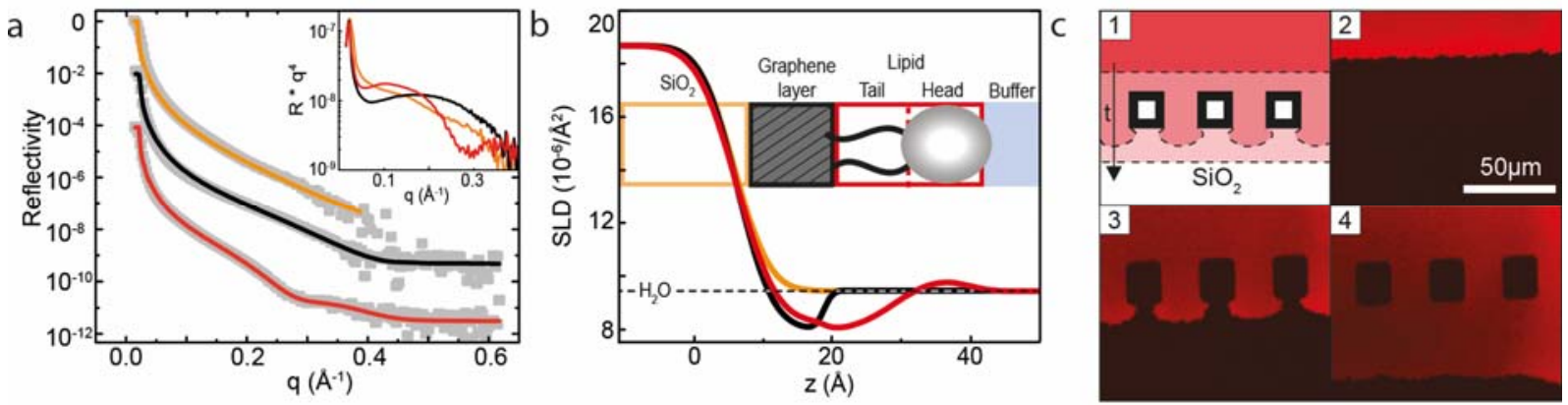

Figure 1: a Normalized X-ray reflectivity data (grey squares) and simulated intensities for the Si wafer with $\mathrm{SiO}_{2}$ layer, after the graphene transfer and with a DOTAP layer, solid lines in orange, black, red, respectively. All measurements were performed in PBS buffer. Data sets are shifted vertically for clarity. The inset shows a superposition of the reflected intensities divided by the Fresnel reflectivity $\mathrm{q}^{-4}$. b Scattering length density depth profiles used to calculate the simulated intensities, same color code as in (a). The dashed line indicates the scattering length density of water. c Spreading of lipids on $\mathrm{SiO}_{2}$ substrate patterned with graphene lines forming square obstacles. The scheme (panel 1) shows the front of the membrane for increasing time (dashed lines). Panels 2-4 are fluorescence microscopy images at different times. Red fluorescence indicates the presence of the $0.5 \%$ Texas Red labeled membrane. Note that the $\mathrm{SiO}_{2}$ patches inside the graphene frames remain uncovered, i.e., the membrane does not cross the graphene barriers. 
Next we evaluate how the monolayer structure influences the capacitance of the graphene. Electrochemical techniques, namely electrical impedance spectroscopy (EIS) and cyclic voltammetry $(\mathrm{CV})$, allow for a detailed electrical characterization of the lipid layer on an electrode $^{68,69}$. Macroscopic graphene electrodes (area $\mathrm{A}=0.023 \mathrm{~cm}^{2}$ ) based on single layer CVD graphene were fabricated as described in the methods section and characterized in a three electrode configuration (the reference electrode is $\mathrm{Ag} / \mathrm{AgCl}$, and the counter electrode is $\mathrm{Pt}$ ) before and after deposition of a DOTAP layer.

a

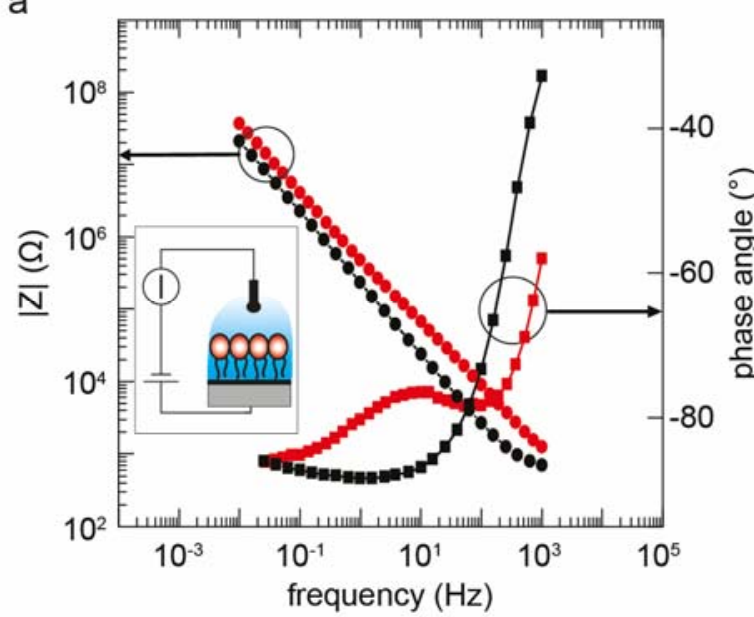

b

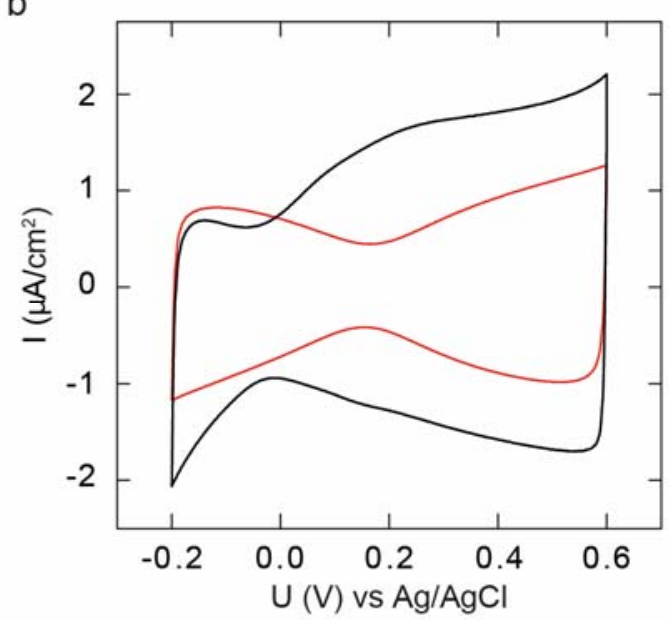

Figure 2: a Electrical impedance showing magnitude (circles) and phase (squares) of graphene before (black) and after formation of a DOTAP layer (red) ( $\mathrm{U}=400 \mathrm{mV}$ vs. $\mathrm{Ag} / \mathrm{AgCl})$. Inset shows a simplified schematic of the measurement configuration, third electrode (counter electrode) is omitted. b Cyclic voltammetry (scan rate $0.5 \mathrm{~V} / \mathrm{s}$ ) of the graphene electrode before (black) and after (red) formation of a DOTAP layer. All measurements were performed in PBS buffer. 
Figure 2a shows the complex electrical impedance $|\mathrm{Z}|$ and phase angle $\varphi$ of a bare graphene electrode (black) and with a DOTAP layer (red). For the bare graphene electrode a quasi-ideal capacitive behavior is observed at frequencies below approximately $100 \mathrm{~Hz}$ in good agreement with previously reported results ${ }^{70}$. The estimated capacitance of the graphene/electrolyte interface estimated from the fitting based on an equivalent circuit (Randles circuit) ranges from $2-4 \mu \mathrm{F} / \mathrm{cm}^{2}$ depending on the applied voltage (see supporting information for a discussion on the voltage dependence of the interface capacitance), in good agreement with literature ${ }^{70-72}$. After deposition of the DOTAP layer, the absolute impedance increases at a fixed frequency, whereas in the phase a second peak arises. This is in qualitative agreement with previous results and can be attributed to a lipid layer on top of the graphene ${ }^{68,73}$. Using EIS, we were able to monitor the kinetics of layer formation, see supporting information Figure S4. The time resolution of EIS is limited to tens of seconds. Similar results were obtained for deposition of 1-palmitoyl-2-oleoylsn-glycero-3-phosphocholine (POPC), a neutral zwitterionic lipid (see supporting information, Figure S3). The EIS data have been modeled using an equivalent circuit (see supporting information) in which the lipid layer capacitance has a value of $3 \mu \mathrm{F} / \mathrm{cm}^{2}$, in line with a vertically compressed lipid monolayer formed on graphene. It is worth mentioning that this value is significantly higher than the expected capacitance of a bilayer $\left(1 \mu \mathrm{F} / \mathrm{cm}^{2}{ }^{68}\right)$. Cyclic voltammetry experiments were performed with bare and lipid covered electrodes. Figure $2 \mathrm{~b}$ shows the result for bare graphene (black) and for a graphene electrode covered with a positively charged DOTAP layer (red). For both measurements, the absence of redox peaks supports the assumption 
of a mainly capacitive interface. The current minimum observed around $-0.05 \mathrm{~V}$ vs $\mathrm{Ag} / \mathrm{AgCl}$ in the case of bare graphene is attributed to the charge neutrality point (CNP) of graphene ${ }^{70}$. The voltage dependent capacitance of the graphene/electrolyte interface, which has been previously discussed in terms of the combined contribution of the quantum capacitance and the electrochemical double layer capacitance ${ }^{74}$, causes the current minimum and the current increase for increasing voltage. After lipid deposition, the current minimum became more pronounced and shifted towards a more positive voltage, $0.175 \mathrm{~V}$. Positively charged lipids near the graphene explain this shift of the CNP as will be discussed in detail using the transistor configuration. Due to the shift of the CNP the effect of the voltage on the graphene/electrolyte capacitance is significantly more pronounced, since it does not interfere with faradaic currents that start to dominate in the negative bias regime. The overall reduction of the current compared to the bare graphene electrode suggests the presence of a second capacitance in series, which is attributed to the lipid layer. Thus, structural and electrical characterization of the lipid graphene interface point towards the formation of a lipid monolayer which mediates the capacitive coupling of the graphene electrolyte interface only weakly. 
a
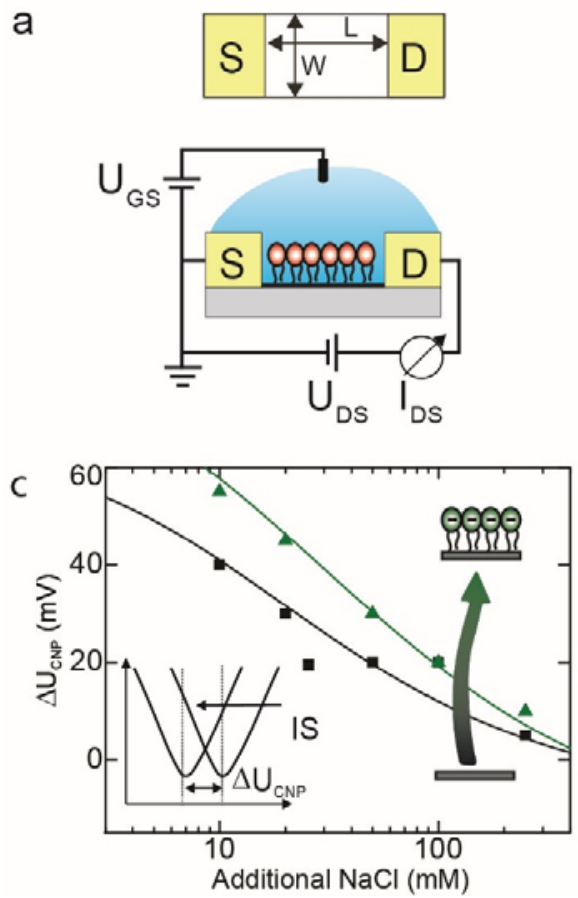
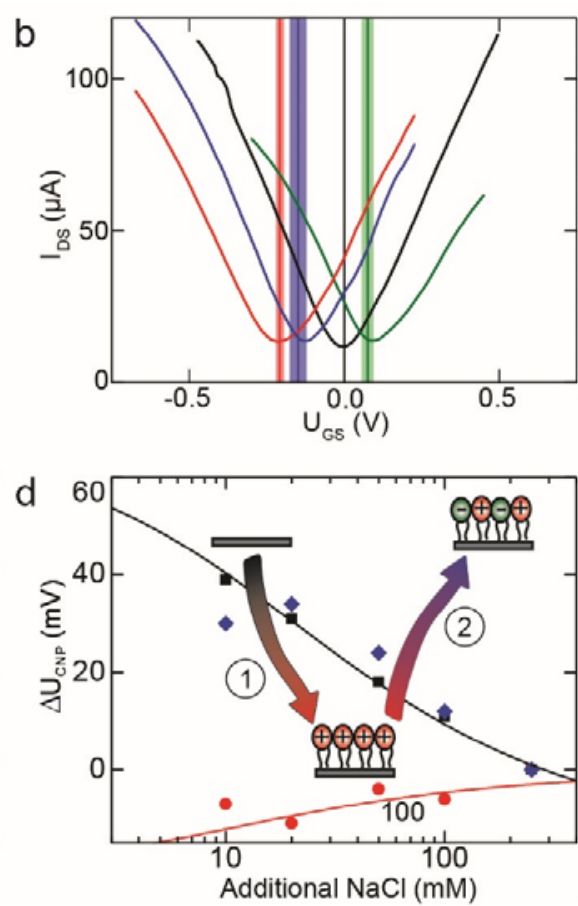

Figure 3: a Upper panel: Top view of graphene SGFET with channel dimensions and source (S) and drain (D) contact. Lower panel: Wiring diagram of a graphene SGFET with lipid monolayer. b Exemplary transistor transfer curves (IDs-UGS) of bare graphene SGFET, and covered with DOTAP, DOTAP/POPG and POPG monolayer shown as black, red, blue, green curve, respectively. The vertical lines indicate the averaged charge neutrality point (CNP) voltage UCNP, i.e., the voltage at minimum current. The colored area indicates the standard deviation. $\mathbf{c}$ Shift of $\mathrm{U}_{\mathrm{CNP}}\left(\triangle \mathrm{U}_{\mathrm{CNP}}\right)$ of a graphene SGFET for increasing ionic strength (IS) in bare state and with a POPG monolayer shown as black squares and green triangles. The sequence of exposure to lipid vesicles for spreading and self-exchange is indicated by the arrow. The inset shows exemplary transistor transfer curves for low and high ion concentration. d Shift of the $U_{C N P}\left(\Delta U_{C N P}\right)$ for a bare transistor, after DOTAP layer deposition and after self-exchange with POPG vesicles, black squares, red circles, and blue diamonds, respectively. Solid lines represent model fits (see main text). All ion sensitivity experiments were performed in 5mM PBS buffer. 
Next, we discuss the experiments performed with micro-scaled graphene solution-gated fieldeffect transistors. Transistors (length $\mathrm{L}=10 \mu \mathrm{m}$, width $\mathrm{W}=20 \mu \mathrm{m}$ ) were fabricated as described in the methods section. The measurement configuration for the graphene SGFETs and their channel dimensions are illustrated in Figure 3a. A typical transfer curve IDs-UGS $\left(U_{D S}=100 \mathrm{mV}\right.$ ) of a graphene SGFET shows the characteristic V-shape curve of ambipolar graphene devices, compare black curve in Figure 3b. The gate voltage was shifted by -UCNP, i.e., the voltage where the current reaches its minimum, of the bare sample. Consequently, in the current minimum of the transfer curve without lipids is at $\mathrm{U}_{\mathrm{GS}}=0 \mathrm{~V}$. The transconductance $\mathrm{g}_{\mathrm{m}}=\partial \mathrm{I}_{\mathrm{DS}} / \partial \mathrm{U}_{\mathrm{GS}}=2 \mathrm{mS} / \mathrm{V}$ is typical for graphene SGFETs ${ }^{1}$. Cationic DOTAP lipids deposited by vesicle fusion on an array (8x8 transistors, see Figure S2) of graphene SGFETs induce a clear shift of the CNP, see Figure $3 \mathrm{~b}$ (red curve), in line with a positive charge brought next to the graphene. The average CNP for different lipid layers on the transistors and their standard deviation is indicated by solid vertical lines and the shaded areas. The average shift (26 transistors) for the DOTAP layer was $\Delta \mathrm{UCNP}_{\mathrm{C}}=-207 \pm 12 \mathrm{mV}$. Similar values were observed for other arrays. This shift is in agreement with the cyclic voltammetry measurements and previously reported values ${ }^{42}$. Note that the sign is inverted due to different grounding convention. A slight reduction in $\mathrm{g}_{\mathrm{m}}$ for both electron and hole regime was observed. Subsequently, anionic 1-palmitoyl-2-oleoyl-sn-glycero-3-phospho(1'-rac-glycerol) (POPG) vesicles were injected in order to allow for self-exchange and rinsed out by deionized water. Afterwards, the transistor curve (blue) shifted towards more positive values, as expected for a negative charge at the graphene surface. An average shift of $\Delta U_{C N P}$ $=-149 \pm 26 \mathrm{mV}$, with respect to the bare graphene, was observed. The exact structure of the 
DOTAP/POPG layer is discussed in the supporting information (see Figure S11). In contrast to the reports of other groups ${ }^{42}$ we could not deposit POPG or other negatively charged lipids on the graphene by vesicle fusion at zero bias. We attribute this to the negative surface charge of graphene ${ }^{1}$ preventing the formation of a negatively charged lipid layer due to electrostatic repulsion ${ }^{75}$. With a negative gate voltage $\left(\mathrm{U}_{\mathrm{GS}}=-300 \mathrm{mV}\right)$, however, it was possible to form an anionic POPG layer on the graphene transistors. This suggests that in the negative bias regime the induced positively charged free carriers can compensate the fixed negative surface charges thus allowing vesicle fusion. Such an example of a graphene SGFET with a POPG layer is shown in Figure $3 \mathrm{~b}$ as green curve. An average shift of the charge neutrality point by $\Delta \mathrm{U}_{\mathrm{CNP}}$ $=+76 \pm 15 \mathrm{mV}$ is observed (25 transistors). The lower absolute shift is in agreement with previously reported results ${ }^{42}$. We found no significant shift of the charge neutrality point for zwitterionic POPC layers (data not shown) which agrees with the results from Ang et al. ${ }^{42}$ but contradicts the results from Wang et al. ${ }^{43}$. Lipids can be removed by immersion in ethanol for several minutes followed by thorough rinsing (see Figure S10 in supporting information).

To assess the surface charge of the lipid layers, we also investigated the ion sensitivity of graphene SGFETs that is influenced by charges at the graphene/electrolyte interface. The ion sensitivity of graphene has been previously discussed in detail in terms of the surface charge at the graphene/electrolyte interface and screening effects ${ }^{1,76}$ (see supporting information for a detailed description of the model ${ }^{77,78}$ ). Figure $3 \mathrm{c}$ shows the normalized shift of the charge neutrality point $\Delta \mathrm{U}_{\mathrm{CNP}}$ as a function of the ion concentration for bare graphene transistors (black) and transistors with a POPG monolayer (green). For bare graphene transistors, we observed a shift of the CNP towards negative voltages (average $-19 \mathrm{mV} / \mathrm{dec}$ ) for increasing sodium chloride concentration. The model correlates the observed shift of the CNP with a surface charge density 
of $-1.7 \mu \mathrm{C} / \mathrm{cm}^{2}$ (solid black line). The POPG monolayer increases the average ion sensitivity to $-35 \mathrm{mV} / \mathrm{dec}$. This is explained by an increase in surface charge from $-1.7 \mu \mathrm{C} / \mathrm{cm}^{2}$ to $-3 \mu \mathrm{C} / \mathrm{cm}^{2}$ in the model (green solid line). Figure 3d shows the normalized position of the charge neutrality point as a function of the salt concentration for bare graphene transistors (black) and transistor with a DOTAP (red) and DOTAP/POPG (blue) layer. The positively charged DOTAP lipids are expected to (over)compensate the negative surface charge and the ion sensitivity is expected to vanish or even inverse its sign. In fact, we observed a slight upshift of the CNP upon increasing salt concentration (average ion sensitivity of $5.6 \mathrm{mV} / \mathrm{dec}$ ) corresponding to a surface charge of $+0.3 \mu \mathrm{C} / \mathrm{cm}^{2}$. This is qualitatively similar to experiments performed at low $\mathrm{pH}$ values where the surface charge of graphene is also inverted ${ }^{76}$. The change in surface charge is in reasonable agreement with the experimentally measured surface charge of positively charged DPTAP lipids $^{79}$; the measured surface charge is significantly lower than a simple estimation based on a positive charge per lipid per $0.9 \mathrm{~nm}^{2}$ since ion-ion interactions have to be considered. Consequently, a hypothetically reduced packing density of DOTAP lipids might have a negligible effect. The self-exchange of cationic DOTAP by anionic POPG lipids (Figure 3d) increases the ion sensitivity again (average $-26 \mathrm{mV} / \mathrm{dec}$ ). This increase indicates the deposition of negative charge at the graphene surface. Possible mechanisms are either the formation of a POPG layer upon the DOTAP layer or the incorporation of POPG lipids into the DOTAP layer. X-ray reflectometry measurements (see Figure S11 in supporting information) confirm that POPG lipids are incorporated into the DOTAP layer, replacing DOTAP lipids. Together with the observed complete recovery of the ion sensitivity, we can conclude that self-exchange occurred in the layer. 
Based on these findings, we propose surface charge and screening effects as an explanation for the changed ion sensitivity of DOTAP layer covered graphene SGFETs. Previous reports suggested complete insulation of the graphene from the electrolyte by the lipid layer ${ }^{43}$ to explain the vanishing ion sensitivity. However, complete insulation is unlikely due to the defectiveness of the supported lipid layer ${ }^{80}$, especially in the case of millimeter-scale transistors used by Wang et al. ${ }^{43}$. Furthermore, such insulation can only explain the reduced ion sensitivity for DOTAP covered transistors, but not the increased sensitivity for POPG covered transistors. In addition, the increased ion sensitivity after incorporation of POPG lipids into the DOTAP layer is a strong argument supporting that the observed ion sensitivity is related to surface charge and screening and not due to the insulation induced by the lipid layer as proposed by Wang et al. ${ }^{81}$.
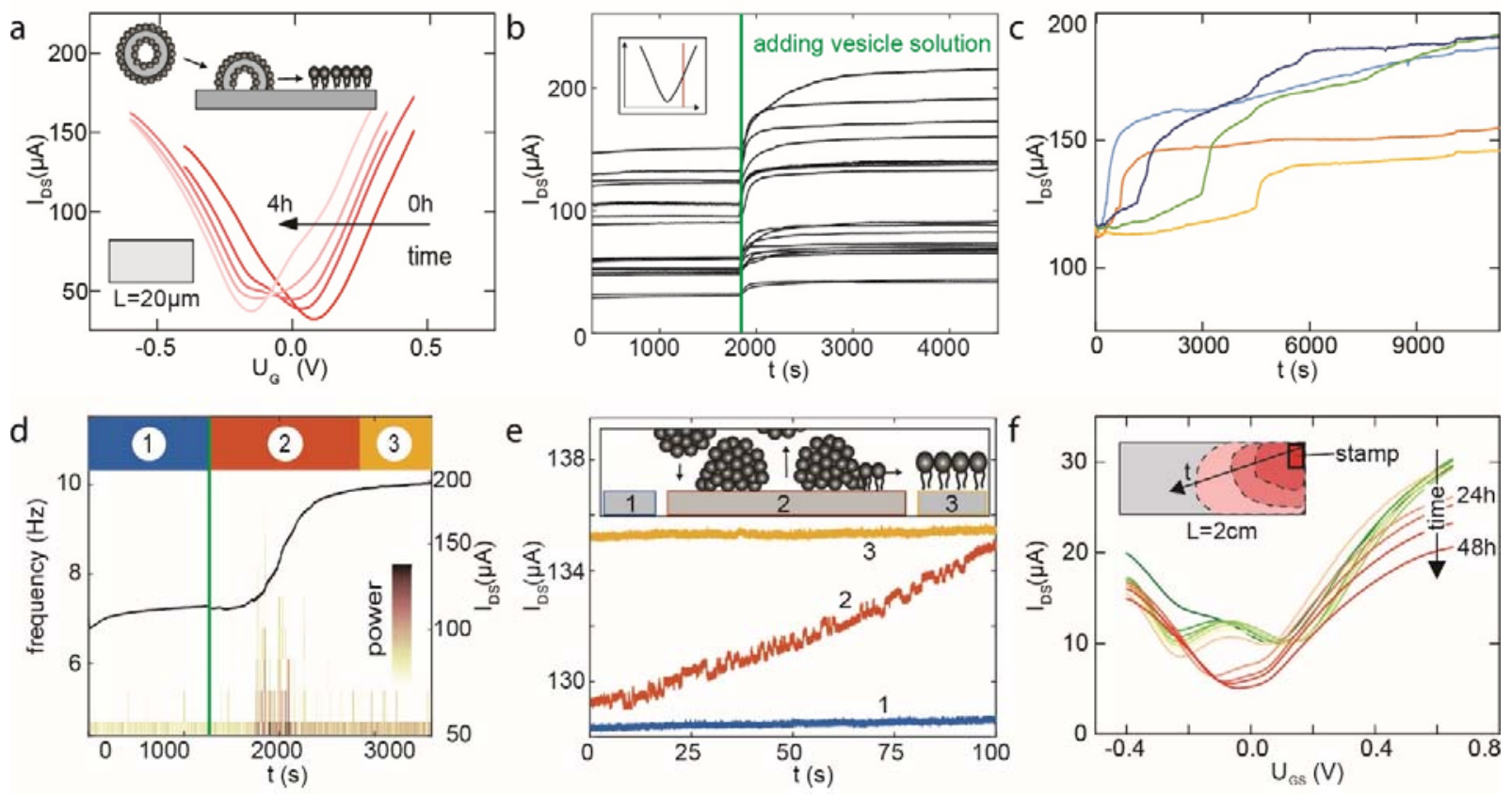
Figure 4: a Evolution of the transistor transfer curves $\left(\mathrm{U}_{\mathrm{DS}}=100 \mathrm{mV}\right)$ with time during formation of a DOTAP lipid layer by vesicle fusion as sketched in the inset. b IDS recording ( $\mathrm{UDS}_{\mathrm{DS}}=100 \mathrm{mV}$, $\mathrm{U}_{\mathrm{GS}}=400 \mathrm{mV}$ as indicated in the inset) of several transistors in parallel analyzing lipid layer formation from freshly extruded vesicles. The time point of adding lipid solution is indicated by green vertical line. c Parallel IDS recording ( $\mathrm{UDS}_{\mathrm{DS}}=100 \mathrm{mV}, \mathrm{UGS}_{\mathrm{GS}}=300 \mathrm{mV}$ ) of lipid layer formation with sonicated lipids stored for several weeks. Lipids were added at $t=0$ s. $\mathbf{d}$ IDs recording of lipid layer formation $\left(\mathrm{UDS}_{\mathrm{DS}}=100 \mathrm{mV}, \mathrm{UGS}_{\mathrm{GS}}=300 \mathrm{mV}\right.$ ) with sonicated lipids stored for several weeks; the time frequency analysis of current signal IDs (topmost curve) in the background. Note, that low frequency $(\mathrm{f}<6 \mathrm{~Hz})$ activity is most pronounced during layer formation, i.e., phase 2 and absent before (1) and after (3). The time point of adding lipid solution is indicated by the green vertical line. e Close-up of IDs recordings from (c) before (blue), during (red) and after lipid layer formation (yellow). Curves were shifted vertically to allow for a better comparison. $\mathbf{f}$ Time evolution of transfer curves $\left(\mathrm{UDS}_{\mathrm{DS}}=100 \mathrm{mV}\right)$ of a macro scale graphene SGFET after stamping of lipids into the corner of the approx. $2 \mathrm{~cm}$ long channel region.

Besides the static characterization of lipid layers on graphene, we investigated the dynamics of the formation of lipid layers on graphene SGFETs. In a first experiment, sonicated DOTAP vesicles were incubated on graphene SGFETs and vesicle fusion was induced by osmotic shock. The transistor curves measured with evolving time (up to 4 hours) after layer formation are shown in Figure 4a. As a result of the presence of the lipid layer, a second CNP starts to form, giving rise to a double dip. Transistor curves with two pronounced CNPs were measured until the first CNP vanished almost completely. After completion of the layer formation only one 
major CNP (pink), shifted with respect to the original one, was measured. A simple three resistor network consisting of a lipid-covered area increasing with time in parallel with an uncovered region and an uncovered region in series can closely reproduce the evolution of the transistor transfer curves (see Figure S8 in supporting information).

In order to better understand the dynamics of lipid formation, we have taken advantage of the sensing capabilities of the transistor configuration, which allow for monitoring of the lipid layer formation with high temporal resolution. To this end, the gate voltage $\mathrm{U}_{\mathrm{GS}}=0.3 \mathrm{~V}$ and drain source voltage $\mathrm{UDS}_{\mathrm{DS}}=100 \mathrm{mV}$ were fixed and the drain-source current was measured while the transistors were exposed to the lipid solution. Figure $4 \mathrm{~b}$ shows the drain-source current as a function of time, recorded in parallel for 23 transistors in one array. The green vertical line indicates the time when the lipid solution (extruded with $200 \mathrm{~nm}$ nominal pore size) is added. Subsequently, the transistor current increases simultaneously for all transistors. This indicates a high tension of the freshly extruded lipids that spread almost instantly on the device surface. As the coverage of the transistor active area with the positively charged lipid layer increases, the drain-source current increases further. The spreading process is qualitatively similar for all transistors, with comparable spreading time, if freshly extruded lipids were used as shown in Figure 4b.

We have also measured sonicated lipids that were stored for several weeks. Figure $4 \mathrm{c}$ shows the recorded current for several transistors; the lipid solution is added at $t=0 \mathrm{~s}$. Opposite to the results obtained with freshly extruded lipids, the drain-source current does not increase instantly, indicating a lower fusiogenity of the stored lipid vesicles. The spreading process occurs at different times for different transistors, and duration and speed vary from transistor to transistor. We attribute the dissimilar response of the transistor to changes in the lipid vesicle with storage 
time $^{82}$. Figure $4 \mathrm{~d}$ shows another experiment with stored lipids using a higher sampling rate. The green vertical line indicates the adding of the lipid solution. Three different time regions, before (1), during (2) and after (3) lipid layer formation are indicated. Interestingly, pronounced current fluctuations are visible during layer formation. To highlight the current fluctuations, a short-time fast Fourier transformation of the recorded current is superimposed in the background of the curve. Increased low frequency components in the signal are observed between $t=30$ minutes up to $t=40$ minutes. A zoom in on the recorded current of the transistor, before (1), during (2) and after (3) lipid layer formation is shown in Figure 4e. During the time of the formation of the lipid layer, "up and down states" in the current are observed. No time correlation between the up and down state of different transistors was observed. We tentatively attribute these states to the adsorption and desorption of lipid aggregates as sketched in the inset of Figure 4e.

Building on the results of the spreading experiment discussed earlier in this paper (see Figure 1c) we also studied lipid spreading from a reservoir on graphene. DOTAP lipids were stamped into one corner of a millimeter-sized graphene SGFET (see the methods section for more information). After stamping, buffer solution was added and the transistor transfer curves were recorded with evolving time. Figure $4 \mathrm{f}$ shows the recorded transistor curves. Already within a very short time after stamping a second minimum in the current-voltage curve can be observed around $-0.2 \mathrm{~V}$. This is expected from a partially lipid covered transistor where a certain part of the graphene has a shifted CNP. With evolving time, the two minima converged two a single, yet broad minimum around $0 \mathrm{~V}$. This indicates increasing coverage of the active area of the graphene transistors with lipids; consequently, this confirms that lipids can spread on graphene, as indicated in the inset in Figure 4f. The overall shift of around $-200 \mathrm{mV}$ is comparable to micro scale SGFETs and CV. The shift of the second minimum from around $-0.2 \mathrm{~V}$ towards $0 \mathrm{~V}$ is 
tentatively attributed to a reduction of the stamped lipids reservoir and a corresponding reduction of positive charges next to the graphene in this area.

\section{CONCLUSION}

In summary, we confirmed the formation of a lipid monolayer on graphene surfaces by X-ray reflectometry. Electrochemical measurements and spreading experiments with lipids on surfaces further corroborate this conclusion. Our findings resolve the structure of lipids on graphene, a prerequisite for the future application of lipid decorated graphene SGFET biosensors. In this line, we have thoroughly discussed the influence of differently charged lipid layers on graphene transistors, especially on their ion sensitivity. The ion sensitivity upon lipid adsorption is modeled by the screening of surface charges. Furthermore, we have demonstrated that graphene transistors can be used to in-situ monitor the formation of lipid layers in real time and with high temporal resolution. Together with recent developments in the fabrication of nanoscale graphene transistors, we envision that this technology has a great potential for studying single vesicle adsorption and desorption. Furthermore, the diagnostics of exosomes, i.e., vesicles secreted by all cells and found in body fluids ${ }^{83}$ holds a great potential. Although the importance of exosomes is known, e.g. ,for non-invasive diagnostics of cancer $^{84}$, new tools to study them must be established. Our work demonstrates that graphene SGFETs can make an important contribution in this field.

\section{EXPERIMENTAL METHODS}

Transistor fabrication: Arrays of 64 transistors were fabricated in one device as follows. Sapphire substrates were pre-patterned with source and drain contacts (titanium/gold, 10/40 nm). 
CVD graphene was grown and transferred using a wet etching transfer as described previously ${ }^{9}$. Graphene was structured by optical photolithography and oxygen plasma etching. A gold overlap layer was evaporated and structured by optical lithography. SU8 GM1040 (Gersteltec, Pully, Switzerland) photoresist, thickness approximately $1 \mu \mathrm{m}$, was spin coated to insulate metal leads. Transistor openings were defined by optical lithography. Samples were wire bonded to a chip carrier. Bond wires were isolated with silicone rubber glue (Scrintec 901, Carl Roth GmbH, Karlsruhe, Germany); a glass ring was mounted on top as electrolyte container.

Transistor measurements: Measurements were performed in a custom-made setup allowing the simultaneous characterization of up to 32 transistors. Briefly, an operational amplifier feedback loop converts the current to a voltage. A National Instruments DAQ Card (National Instruments, Austin, USA) records the voltages. Gate and drain source voltage was applied with the DAQ card for transistor characterization. Measurements were performed in a two-electrode configuration (Ag/AgCl FLEXREF electrode, World precision instrument, Berlin, Germany), with the drain contact being set to ground. This is contrary to the standard electrochemistry convention.

Electrochemistry: Graphene was transferred to glass substrates and contacted with a wire using silver paste. Silver paste and wire were covered with silicone rubber (Scrintec 901). An electrolyte container (glass cylinder) was mounted on top. Measurements were performed using a potentiostat (Gamry instruments, Warminster, Pennsylvania, USA) in PBS Dulbecco in a threeelectrode configuration. A platinum counter electrode and $\mathrm{Ag} / \mathrm{AgCl}$ reference electrode (FlexRef) were used.

Lipid preparation: All non-labeled lipids were purchased from AvantiPolar Lipids (Alabaster, Alabama, USA); Texas Red DHPE was purchased from ThermoFisher (Waltham, 
Massachusetts, USA). To prepare lipid vesicles, the desired amount of lipids dissolved in chloroform was put in a glass vial in desired mixture. The chloroform was then evaporated under nitrogen flow and the vial stored in vacuum overnight. The dried lipids then were suspended to $0.5 \mathrm{mg} / \mathrm{ml}$ in Dulbecco PBS (Sigma Aldrich, Taufkirchen, Germany). Before use they were diluted with Dulbecco's PBS (1:20).

Extrusion: The suspension was passed 11 times through a polycarbonate filter with pores of a size of $100 \mathrm{~nm}, 200 \mathrm{~nm}$ or $1 \mu \mathrm{m}$ (AVANTI) to produce unilamellar vesicles ${ }^{85}$.

Tip sonication: The suspension was tip sonicated (Bandelin electronic Berlin, Berlin, Germany) for 10 minutes with $60 \%$ of maximum power. Suspension was centrifuged for 3 minutes with $10000 \mathrm{RCF}$ and pipetted off afterwards to get rid of eventual metal swarf.

The results of the dynamic light scattering (DLS) measurements of DOTAP vesicles prepared with the presented methods and used in this work are shown in the supporting information (Table S2).

\section{Lipid layer formation}

Layer formation was achieved by immersion of the substrate in a vesicle-containing solution. Osmotic shock ${ }^{86}$, i.e. exchange of the buffer solution with DI water was also tried, but found to have no advantage. After layer formation the buffer was changed several times to remove residual SUVs. Air exposure was avoided. Layer formation was carried out at room temperature. For X-ray reflectometry experiments the samples were incubated overnight, more details in Xray reflectivity measurements. For electrochemistry experiments the samples were incubated overnight and measurements were performed in between to monitor bilayer formation. For transistor measurements, when the lipid layer formation was not monitored, the samples were 
incubated with SUVs for approx. one hour. The samples were not immersed in water before layer formation. However, they were exposed to buffer before to perform a characterization prior to the lipid deposition.

\section{Self spreading of lipid layer}

$7.5 \mathrm{mg}$ of lipids (99.5\% POPC and $0.05 \%$ TexasRed DHPE) were mixed and dried as for vesicle preparation. After drying the lipids were dissolved in $1 \mathrm{ml}$ isopropyl alcohol and a few $\mu \mathrm{ml}$ were placed on a PDMS stamp (made of Sylgard Elastomer 184). Stamps were dried overnight in a vacuum chamber. Lipids were stamped on target substrate with gentle pressure. In a final step, PBS Dulbecco buffer solution was added to start the spreading process.

X-ray reflectivity measurements: X-ray reflectivity measurements were performed at the custom-built molybdenum-anode-based in-house reflectometer. It consists of a molybdenum line focus X-ray tube (Seifert DX MO 10x0.15, GE, Boston, USA). A Goebel multilayer mirror (Rigaku, Tokyo, Japan) delivers monochromatic beam with energy of $17.4 \mathrm{keV}$. The beam size is $8 \mathrm{~mm} \times 0.35 \mathrm{~mm}$ after collimation. Data is recorded by a 1D NaI detector (Seifert). Experiments were performed as $\theta-2 \theta$ scans of sample and detector angle, background measurements with an offset for $\theta$ of $-0.05^{\circ}$ and corrected for by subtraction. To take account for the effective beam height at low angles, geometrical corrections were performed ${ }^{87}$. The intensity of all measurements was normalized to 1 and converted to momentum transfer by $q=4 \pi / \lambda \sin (2 \theta / 2)$.

Measurements were performed in a slightly modified version of a sample chamber we previously reported about ${ }^{88}$. Every wafer (purchased from MicroChemicals GmbH, Ulm, Germany, with an oxide layer of $200 \mathrm{~nm}$ and a size of $15 \times 20 \times 0.7 \mathrm{~mm}^{3}$ ) was measured before and after graphene transfer. After gluing the wafer to the chamber (Microset 101RF, Microset, Leicestershire, UK), the chamber was filled with Dulbecco's PBS and experiment started. After 
the measurement of the graphene layer, vesicle solution $(1 \mathrm{mg}: 20 \mathrm{ml})$ was injected into the chamber and incubated over night. This allows the lipid vesicles to adhere to the surface. The vesicles were then ruptured by osmotic shock and afterwards flushed intensively with Dulbecco's PBS to remove lipid aggregates / eventual multilayer.

X-ray reflectivity data modelling: The SLD profiles were modeled using MOTOFIT. The minimal model to reproduce the data is always chosen. The program uses $\mathrm{n}$ numbers of discrete layers with constant SLD and thickness, the so-called boxes, to describe the system. At the interface of two adjoining boxes an error function takes the roughness into account. The program converts the modeled SLD profile to a theoretical reflectivity curve using the Abeles formalism. This theoretical reflectivity curve is then fitted to the data.

\section{ASSOCIATED CONTENT}

\section{Supporting information}

The Supporting Information is available free of charge on the ACS Publications website.

Schematics of device fabrication, optical images of SGFET array, EIS data from POPC layer and DOTAP layer formation, Schematic of graphene/electrolyte interface capacitance, Modelling of EIS data, Noise measurements of DOTAP layers on graphene SGFETs, Models for lipid spreading and SGFET ion sensitivity, Ethanol cleaning of transistor, Reflectometry data and modelling of DOTAP/POPG monolayer, Fitting parameter for modelling of reflectivity data, Average diameters for lipid vesicles used in this work measured by dynamic light scattering. 


\section{AUTHOR INFORMATION}

\section{Corresponding Author}

J.A.G and B.N.

\section{Author Contributions}

B.M.B., P.B., B.N. and J.A.G. designed the experiment, J.A.G and B.N. supervised the experiments. B.M.B. and S.D. grew and transferred graphene. P.B. prepared lipid vesicle solutions. B.M.B. fabricated the samples for transistor, electrochemical and X-ray reflectometry measurements. B.M.B. performed and analyzed transistor and spreading experiments. B.M.B. performed electrochemical measurements and analyzed them with S.D.. P.B. performed and analyzed X-ray reflectometry experiments. B.M.B., P.B., B.N. and J.A.G. interpreted the results of the experiments and co-wrote the paper. All authors have given approval to the final version of the manuscript.

\section{Notes}

The authors declare no competing financial interests.

\section{ACKNOWLEDGMENT}

This work has been funded by the European Union's Horizon 2020 research and innovation programme under grant agreement No 696656 (Graphene Flagship), BMBF (Project 05K13WM1) and SFB1032 (Project A7). The ICN2 is supported by the Severo Ochoa programme of the Spanish Ministry of Economy, Industry and Competitiveness (MINECO, grant no. SEV-2013-0295). We thank Erich Sackmann, Joachim Rädler, Peter Cevc and Babak Sanii for fruitful discussions. The authors thank Stefan Holler for test measurements, Tobias Wimmer 
and Peter Knecht for the help with the transistor measurements and Florian Ehrat for the help with the DSL measurements. 


\section{References}

(1) Hess, L. H., Seifert, M., Garrido, J. A. Graphene Transistors for Bioelectronics. Proc. IEEE 2013, 101, 1780-1792.

(2) Fu, W., El Abbassi, M., Hasler, T., Jung, M., Steinacher, M., Calame, M., Schönenberger, C., Puebla-Hellmann, G., Hellmüller, S., Ihn, T., Wallraff, A. Electrolyte gate dependent high-frequency measurement of graphene field-effect transistor for sensing applications. Appl. Phys. Lett. 2014, 104, 13102.

(3) Fu, W., Feng, L., Mayer, D., Panaitov, G., Kireev, D., Offenhäusser, A., Krause, H.-J. Electrolyte-Gated Graphene Ambipolar Frequency Multipliers for Biochemical Sensing. Nano Lett. 2016.

(4) Wang, X., Zhi, L., Müllen, K. Transparent, Conductive Graphene Electrodes for DyeSensitized Solar Cells. Nano Lett. 2008, 8, 323-327.

(5) Blake, P., Brimicombe, P. D., Nair, R. R., Booth, T. J., Jiang, D., Schedin, F., Ponomarenko, L. A., Morozov, S. V., Gleeson, H. F., Hill, E. W., Geim, A. K., Novoselov, K. S. Graphene-based liquid crystal device. Nano letters 2008, 8, 1704-1708.

(6) Park, S. J., Kwon, O. S., Lee, S. H., Song, H. S., Park, T. H., Jang, J. Ultrasensitive flexible graphene based field-effect transistor (FET)-type bioelectronic nose. Nano letters 2012, 12, 5082-5090.

(7) Cohen-Karni, T., Qing, Q., Li, Q., Fang, Y., Lieber, C. M. Graphene and nanowire transistors for cellular interfaces and electrical recording. Nano letters 2010, 10, 1098-1102.

(8) Hess, L. H., Jansen, M., Maybeck, V., Hauf, M. V., Seifert, M., Stutzmann, M., Sharp, I. D., Offenhäusser, A., Garrido, J. A. Graphene Transistor Arrays for Recording Action Potentials from Electrogenic Cells. Adv. Mater. 2011, 23, 5045-5049.

(9) Blaschke, B. M., Lottner, M., Drieschner, S., Calia, A. B., Stoiber, K., Rousseau, L., Lissourges, G., Garrido, J. A. Flexible graphene transistors for recording cell action potentials. 2D Mater. 2016, 3, 25007.

(10) Veliev, F., Han, Z., Kalita, D., Briançon-Marjollet, A., Bouchiat, V., Delacour, C. Recording Spikes Activity in Cultured Hippocampal Neurons Using Flexible or Transparent Graphene Transistors. Frontiers in neuroscience 2017, 11, 466.

(11) Blaschke, B. M., Tort-Colet, N., Guimerà-Brunet, A., Weinert, J., Rousseau, L., Heimann, A., Drieschner, S., Kempski, O., Villa, R., Sanchez-Vives, M. V., Garrido, J. A. Mapping brain activity with flexible graphene micro-transistors. 2D Mater. 2017, 4, 25040.

(12) Hébert, C., Masvidal-Codina, E., Suarez-Perez, A., Calia, A. B., Piret, G., GarciaCortadella, R., Illa, X., Del Corro Garcia, E., La Cruz Sanchez, J. M. de, Casals, D. V., Prats-Alfonso, E., Bousquet, J., Godignon, P., Yvert, B., Villa, R., Sanchez-Vives, M. V., Guimerà-Brunet, A., Garrido, J. A. Flexible Graphene Solution-Gated Field-Effect Transistors: Efficient Transducers for Micro-Electrocorticography. Adv. Funct. Mater. 2017, 453, 1703976.

(13) Hess, L. H., Lyuleeva, A., Blaschke, B. M., Sachsenhauser, M., Seifert, M., Garrido, J. A., Deubel, F. Graphene Transistors with Multifunctional Polymer Brushes for Biosensing Applications. ACS Appl. Mater. Interfaces 2014, 6, 9705-9710.

(14) Ping, J., Vishnubhotla, R., Vrudhula, A., Johnson, A. T. C. Scalable Production of HighSensitivity, Label-Free DNA Biosensors Based on Back-Gated Graphene Field Effect Transistors. ACS Nano 2016, 10, 8700-8704. 
(15) Gao, N., Gao, T., Yang, X., Dai, X., Zhou, W., Zhang, A., Lieber, C. M. Specific detection of biomolecules in physiological solutions using graphene transistor biosensors. Proceedings of the National Academy of Sciences of the United States of America 2016, 113, 14633-14638.

(16) Hirtz, M., Oikonomou, A., Georgiou, T., Fuchs, H., Vijayaraghavan, A. Multiplexed biomimetic lipid membranes on graphene by dip-pen nanolithography. Nature communications 2013, 4, 2591.

(17) Sackmann, E. Supported membranes: Scientific and practical applications. Science 1996, 271, 43-48.

(18) Nissen, J., Jacobs, K., Radler, J. O. Interface dynamics of lipid membrane spreading on solid surfaces. Physical review letters 2001, 86, 1904-1907.

(19) Brüggemann, D., Frohnmayer, J. P., Spatz, J. P. Model systems for studying cell adhesion and biomimetic actin networks. Beilstein journal of nanotechnology 2014, 5, 1193-1202.

(20) Loose, M., Schwille, P. Biomimetic membrane systems to study cellular organization. Journal of structural biology 2009, 168, 143-151.

(21) Castellana, E. T., Cremer, P. S. Solid supported lipid bilayers: From biophysical studies to sensor design. Surface Science Reports 2006, 61, 429-444.

(22) Magliulo, M., Mallardi, A., Mulla, M. Y., Cotrone, S., Pistillo, B. R., Favia, P., VikholmLundin, I., Palazzo, G., Torsi, L. Electrolyte-gated organic field-effect transistor sensors based on supported biotinylated phospholipid bilayer. Advanced materials (Deerfield Beach, Fla.) 2013, 25, 2090-2094.

(23) Fairn, G. D., Grinstein, S. A One-Sided Signal. Science (New York, N.Y.) 2008, 320, 458.

(24) Fritz, K., Fritz, G., Windschiegl, B., Steinem, C., Nickel, B. Arrangement of Annexin A2 tetramer and its impact on the structure and diffusivity of supported lipid bilayers. Soft matter 2010, 6, 4084.

(25) Khodagholy, D., Rivnay, J., Sessolo, M., Gurfinkel, M., Leleux, P., Jimison, L. H., Stavrinidou, E., Herve, T., Sanaur, S., Owens, R. M., Malliaras, G. G. High transconductance organic electrochemical transistors. Nature communications 2013, 4, 2133.

(26) Khodagholy, D., Rivnay, J., Sessolo, M., Gurfinkel, M., Leleux, P., Jimison, L. H., Stavrinidou, E., Herve, T., Sanaur, S., Owens, R. M., Malliaras, G. G. High transconductance organic electrochemical transistors. Nature communications 2013, 4, 2133.

(27) Werkmeister, F. X., Nickel, B. A. Fast detection of blood gases by solution gated organic field effect transistors. Organic Electronics 2016, 39, 113-117.

(28) Zhang, Y., Inal, S., Hsia, C.-Y., Ferro, M., Ferro, M., Daniel, S., Owens, R. M. Supported Lipid Bilayer Assembly on PEDOT: PSS Films and Transistors. Adv. Funct. Mater. 2016, 26, 7304-7313.

(29) Steinhoff, G., Baur, B., Wrobel, G., Ingebrandt, S., Offenhäusser, A., Dadgar, A., Krost, A., Stutzmann, M., Eickhoff, M. Recording of cell action potentials with AlGaN/GaN fieldeffect transistors. Appl. Phys. Lett. 2005, 86, 33901.

(30) Ang, P. K., Loh, K. P., Wohland, T., Nesladek, M., van Hove, E. Supported Lipid Bilayer on Nanocrystalline Diamond: Dual Optical and Field-Effect Sensor for Membrane Disruption. Adv. Funct. Mater. 2009, 19, 109-116. 
(31) Dankerl, M., Eick, S., Hofmann, B., Hauf, M., Ingebrandt, S., Offenhäusser, A., Stutzmann, M., Garrido, J. A. Diamond Transistor Array for Extracellular Recording From Electrogenic Cells. Adv. Funct. Mater. 2009, 19, 2915-2923.

(32) Cui, Y., Wei, Q., Park, H., Lieber, C. M. Nanowire nanosensors for highly sensitive and selective detection of biological and chemical species. Science 2001, 293, 1289-1292.

(33) Allen, B. L., Kichambare, P. D., Star, A. Carbon Nanotube Field-Effect-Transistor-Based Biosensors. Adv. Mater. 2007, 19, 1439-1451.

(34) Heller, I., Janssens, A. M., Männik, J., Minot, E. D., Lemay, S. G., Dekker, C. Identifying the mechanism of biosensing with carbon nanotube transistors. Nano Lett. 2008, 8, 591595.

(35) Patolsky, F., Zheng, G., Lieber, C. M. Nanowire sensors for medicine and the life sciences. Nanomedicine (London, England) 2006, 1, 51-65.

(36) Gao, X. P. A., Zheng, G., Lieber, C. M. Subthreshold regime has the optimal sensitivity for nanowire FET biosensors. Nano letters 2010, 10, 547-552.

(37) Huang, S.-C. J., Artyukhin, A. B., Misra, N., Martinez, J. A., Stroeve, P. A., Grigoropoulos, C. P., Ju, J.-W. W., Noy, A. Carbon nanotube transistor controlled by a biological ion pump gate. Nano letters 2010, 10, 1812-1816.

(38) Misra, N., Martinez, J. A., Huang, S.-C. J., Wang, Y., Stroeve, P., Grigoropoulos, C. P., Noy, A. Bioelectronic silicon nanowire devices using functional membrane proteins. Proceedings of the National Academy of Sciences of the United States of America 2009, 106, 13780-13784.

(39) Martinez, J. A., Misra, N., Wang, Y., Stroeve, P., Grigoropoulos, C. P., Noy, A. Highly efficient biocompatible single silicon nanowire electrodes with functional biological pore channels. Nano Lett. 2009, 9, 1121-1126.

(40) Williams, E. H., Ha, J.-Y., Juba, M., Bishop, B., Krylyuk, S., Motayed, A., Rao, M. V., Schreifels, J. A., Davydov, A. V. Real-time electrical detection of the formation and destruction of lipid bilayers on silicon nanowire devices. Sensing and Bio-Sensing Research 2015, 4, 103-108.

(41) Fu, W., El Abbassi, M., Hasler, T., Jung, M., Steinacher, M., Calame, M., Schönenberger, C., Puebla-Hellmann, G., Hellmüller, S., Ihn, T., Wallraff, A. Electrolyte gate dependent high-frequency measurement of graphene field-effect transistor for sensing applications. Appl. Phys. Lett. 2014, 104, 13102.

(42) Ang, P. K., Jaiswal, M., Lim, C. H. Y. X., Wang, Y., Sankaran, J., Li, A., Lim, C. T., Wohland, T., Barbaros, O., Loh, K. P. A bioelectronic platform using a graphene-lipid bilayer interface. ACS Nano 2010, 4, 7387-7394.

(43) Wang, Y. Y., Pham, T. D., Zand, K., Li, J., Burke, P. J. Charging the quantum capacitance of graphene with a single biological ion channel. ACS Nano 2014, 8, 4228-4238.

(44) Tabaei, S. R., Ng, W. B., Cho, S.-J., Cho, N.-J. Controlling the Formation of Phospholipid Monolayer, Bilayer, and Intact Vesicle Layer on Graphene. ACS applied materials \& interfaces 2016, 8, 11875-11880.

(45) Hirtz, M., Oikonomou, A., Georgiou, T., Fuchs, H., Vijayaraghavan, A. Multiplexed biomimetic lipid membranes on graphene by dip-pen nanolithography. Nature communications 2013, 4, 2591.

(46) Richter, R. P., Bérat, R., Brisson, A. R. Formation of solid-supported lipid bilayers: an integrated view. Langmuir the ACS journal of surfaces and colloids 2006, 22, 3497-3505. 
(47) Soung, Y. H., Ford, S., Zhang, V., Chung, J. Exosomes in Cancer Diagnostics. Cancers 2017, 9.

(48) Nissen, J., Gritsch, S., Wiegand, G., Rädler, J. O. Wetting of phospholipid membranes on hydrophilic surfaces - Concepts towards self-healing membranes. Eur. Phys. J. B 1999, 10, 335-344.

(49) Kim, J., Cote, L. J., Kim, F., Huang, J. Visualizing graphene based sheets by fluorescence quenching microscopy. Journal of the American Chemical Society 2010, 132, 260-267.

(50) Swathi, R. S., Sebastian, K. L. Resonance energy transfer from a dye molecule to graphene. The Journal of chemical physics 2008, 129, 54703.

(51) Sanii, B., Parikh, A. N. Surface-energy dependent spreading of lipid monolayers and bilayers. Soft matter 2007, 3, 974.

(52) Keller, C. A., Kasemo, B. Surface Specific Kinetics of Lipid Vesicle Adsorption Measured with a Quartz Crystal Microbalance. Biophysical Journal 1998, 75, 1397-1402.

(53) S.J. Johnson, T.M. Bayerl, D.C. McDermott, G.W. Adam, A.R. Rennie, R.K. Thomas, E. Sackmann. Structure of an adsorbed dimyristoylphosphatidylcholine bilayer measured with specular reflection of neutrons. Biophysical Journal 1991, 289-294.

(54) Vogel, M., Münster, C., Fenzl, W., Salditt, T. Thermal unbinding of highly oriented phospholipid membranes. Physical review letters 2000, 84, 390-393.

(55) Tanaka, M., Tutus, M., Kaufmann, S., Rossetti, F. F., Schneck, E., Weiss, I. M. Native supported membranes on planar polymer supports and micro-particle supports. Journal of structural biology 2009, 168, 137-142.

(56) Wacklin, H. P. Neutron reflection from supported lipid membranes. Current Opinion in Colloid \& Interface Science 2010, 15, 445-454.

(57) Gerelli, Y., Porcar, L., Lombardi, L., Fragneto, G. Lipid exchange and flip-flop in solid supported bilayers. Langmuir the ACS journal of surfaces and colloids 2013, 29, 1276212769.

(58) Hertrich, S., Stetter, F., Rühm, A., Hugel, T., Nickel, B. Highly hydrated deformable polyethylene glycol-tethered lipid bilayers. Langmuir the ACS journal of surfaces and colloids 2014, 30, 9442-9447.

(59) Jens Als-Nielsen and Des McMorrow, Ed. Elements of modern X-ray physics; John Wiley \& Sons, 2011.

(60) Pershan, P. S. X-ray or neutron reflectivity: Limitations in the determination of interfacial profiles. Phys. Rev. E 1994, 2369-2373.

(61) Nelson, A. Co-refinement of multiple-contrast neutron/X-ray reflectivity data using MOTOFIT. J. Appl. Crystallogr. 2006, 39, 273-276.

(62) Lima, L. M. C., Fu, W., Jiang, L., Kros, A., Schneider, G. F. Graphene-stabilized lipid monolayer heterostructures: A novel biomembrane superstructure. Nanoscale 2016, 8, 18646-18653.

(63) Dan, Y., Lu, Y., Kybert, N. J., Luo, Z., Johnson, A. T. C. Intrinsic response of graphene vapor sensors. Nano letters 2009, 9, 1472-1475.

(64) Nagle, J. F., Tristram-Nagle, S. Structure of lipid bilayers. Biochimica et Biophysica Acta (BBA) - Reviews on Biomembranes 2000, 1469, 159-195.

(65) Raedler, J., Strey, H., Sackmann, E. Phenomenology and Kinetics of Lipid Bilayer Spreading on Hydrophilic Surfaces. Langmuir 1995, 11, 4539-4548.

(66) Li, W., Chung, J. K., Lee, Y. K., Groves, J. T. Graphene-Templated Supported Lipid Bilayer Nanochannels. Nano Lett. 2016, 16, 5022-5026. 
(67) Hong, G., Han, Y., Schutzius, T. M., Wang, Y., Pan, Y., Hu, M., Jie, J., Sharma, C. S., Müller, U., Poulikakos, D. On the Mechanism of Hydrophilicity of Graphene. Nano letters 2016, 16, 4447-4453.

(68) Wiegand, G., Arribas-Layton, N., Hillebrandt, H., Sackmann, E., Wagner, P. Electrical Properties of Supported Lipid Bilayer Membranes. J. Phys. Chem. B 2002, 106, 4245-4254.

(69) Lingler, S., Rubinstein, I., Knoll, W., Offenhäusser, A. Fusion of Small Unilamellar Lipid Vesicles to Alkanethiol and Thiolipid Self-Assembled Monolayers on Gold. Langmuir the ACS journal of surfaces and colloids 1997, 13, 7085-7091.

(70) Drieschner, S., Guimerà, A., Cortadella, R. G., Viana, D., Makrygiannis, E., Blaschke, B. M., Vieten, J., Garrido, J. A. Frequency response of electrolyte-gated graphene electrodes and transistors. J. Phys. D: Appl. Phys. 2017, 50, 95304.

(71) Dankerl, M., Hauf, M. V., Lippert, A., Hess, L. H., Birner, S., Sharp, I. D., Mahmood, A., Mallet, P., Veuillen, J.-Y., Stutzmann, M., Garrido, J. A. Graphene Solution-Gated FieldEffect Transistor Array for Sensing Applications. Adv. Funct. Mater. 2010, 20, 3117-3124.

(72) Ji, H., Zhao, X., Qiao, Z., Jung, J., Zhu, Y., Lu, Y., Zhang, L. L., MacDonald, A. H., Ruoff, R. S. Capacitance of carbon-based electrical double-layer capacitors. Nat Comms 2014, 5, 3317.

(73) Schubert, T., Steinhoff, G., Ribbeck, H.-G. von, Stutzmannn, M., Eickhoff, M., Tanaka, M. Gallium nitride electrodes for membrane-based electrochemical biosensors. The European physical journal. E, Soft matter 2009, 30, 233-238.

(74) Dankerl, M., Hauf, M. V., Lippert, A., Hess, L. H., Birner, S., Sharp, I. D., Mahmood, A., Mallet, P., Veuillen, J.-Y., Stutzmann, M., Garrido, J. A. Graphene Solution-Gated FieldEffect Transistor Array for Sensing Applications. Adv. Funct. Mater. 2010, 20, 3117-3124.

(75) Cremer, P. S., Boxer, S. G. Formation and Spreading of Lipid Bilayers on Planar Glass Supports. J. Phys. Chem. B 1999, 103, 2554-2559.

(76) Heller, I., Chatoor, S., Männik, J., Zevenbergen, M. A. G., Dekker, C., Lemay, S. G. Influence of electrolyte composition on liquid-gated carbon nanotube and graphene transistors. Journal of the American Chemical Society 2010, 132, 17149-17156.

(77) Männik, J., Heller, I., Janssens, A. M., Lemay, S. G., Dekker, C. Charge noise in liquidgated single-wall carbon nanotube transistors. Nano Lett. 2008, 8, 685-688.

(78) Härtl, A., Garrido, J. A., Nowy, S., Zimmermann, R., Werner, C., Horinek, D., Netz, R., Stutzmann, M. The ion sensitivity of surface conductive single crystalline diamond. Journal of the American Chemical Society 2007, 129, 1287-1292.

(79) Klausen, L. H., Fuhs, T., Dong, M. Mapping surface charge density of lipid bilayers by quantitative surface conductivity microscopy. Nat Comms 2016, 7, 12447.

(80) Cevc, G. Membrane electrostatics. Biochimica et biophysica acta 1990, 1031, 311-382.

(81) Wang, X., Zhi, L., Müllen, K. Transparent, conductive graphene electrodes for dyesensitized solar cells. Nano letters 2008, 8, 323-327.

(82) Cho, N.-J., Hwang, L. Y., Solandt, J. J. R., Frank, C. W. Comparison of Extruded and Sonicated Vesicles for Planar Bilayer Self-Assembly. Materials (Basel, Switzerland) 2013, 6, 3294-3308.

(83) Vlassov, A. V., Magdaleno, S., Setterquist, R., Conrad, R. Exosomes: Current knowledge of their composition, biological functions, and diagnostic and therapeutic potentials. Biochimica et biophysica acta 2012, 1820, 940-948.

(84) Melo, S. A., Luecke, L. B., Kahlert, C., Fernandez, A. F., Gammon, S. T., Kaye, J., LeBleu, V. S., Mittendorf, E. A., Weitz, J., Rahbari, N., Reissfelder, C., Pilarsky, C., Fraga, M. F., 
Piwnica-Worms, D., Kalluri, R. Glypican-1 identifies cancer exosomes and detects early pancreatic cancer. Nature 2015, 523, 177-182.

(85) MacDonald, R. C., MacDonald, R. I., Menco, B. P.M., Takeshita, K., Subbarao, N. K., Hu, L.-r. Small-volume extrusion apparatus for preparation of large, unilamellar vesicles. Biochimica et Biophysica Acta (BBA) - Biomembranes 1991, 1061, 297-303.

(86) Cohen, F. S., Akabas, M. H., Finkelstein, A. Osmotic swelling of phospholipid vesicles causes them to fuse with a planar phospholipid bilayer membrane. Science 1982, 458-460.

(87) Salah, F., Harzallah, B., van der Lee, A. Data reduction practice in X-ray reflectometry. $J$ Appl Crystallogr 2007, 40, 813-819.

(88) Reich, C., Hochrein, M. B., Krause, B., Nickel, B. A microfluidic setup for studies of solidliquid interfaces using $\mathrm{x}$-ray reflectivity and fluorescence microscopy. Review of Scientific Instruments 2005, 76, 95103. 